\title{
OFFICERS OF THE ASSOCIATION
}

1924 Temporary Chairman-V. V. Anderson, M.D.

Temporary Secretary-Karl A. Menninger, M.D.

\section{President}

1924-26 William Healy, M.D.

*1926-27 Herman M. Adler, M.D.

1927-28 Karl A. Menninger, M.D.

1928-30 Lawson G. Lowrey, M.D.

1930-31 David M. Levy, M.D.

1931-32 Augusta F. Bronner, Ph.D.

*1932-33 Ira S. Wile, M.D.

1933-34 Frederick H. Allen, M.D.

1934-35 George S. Stevenson, M.D.

1935-36 Ralph P. Truitt, M.D.

1936-37 Edgar A. Doll, Ph.D.

1937-38 George J. Mohr, M.D.

1938-39 Frank J. O'Brien, M.D.

1939-40 Samuel W. Hartwell, M.D.

1940-41 Paul L. Schroeder, M.D.

*1941-42 Jacob Kasanin, M.D.

1942-43 Henry C. Schumacher, M.D.

1943-44 George H. Preston, M.D.

1944-46 Norvelle C. LaMar, M.D.

1946-47 Spafford Ackerly, M.D.

1947-48 Milton E. Kirkpatrick, M.D.

Vice President

*1924-26 Arnold Jacoby, M.D.

1926-27 George H. Reeve, M.D.

*1927-28 Ira S. Wile, M.D.

1928-30 Augusta F. Bronner, Ph.D.

1930-31 Edgar A. Doll, Ph.D.

1931-32 Ralph P. Truitt, M.D.

1932-33 Frederick H. Allen, M.D.

1933-34 Bertha C. Reynolds

1934-35 H. Meltzer, Ph.D.

1935-36 Willard C. Olson, Ph.D.

1936-37 Henry C. Schumacher, M.D.

1937-38 Elma Olson

1938-39 Almena Dawley

1939-40 E. K. Wickman

1940-41 Helen L. Witmer, Ph.D.

1941-42 Carl R. Rogers, Ph.D.

1942-43 Simon H. Tulchin

1943-44 S. J. Beck, Ph.D.

1944-46 Lawrence K. Frank

1946-47 Phyllis Blanchard, Ph.D.

1947-48 Marian McBee

\section{Secretary-Treasurer}

1924-26 Karl A. Menninger, M.D.

*1926-27 Arnold Jacoby, M.D.

1927 Karl A. Menninger, M.D. Acting

1927-34 George S. Stevenson, M.D.

1934-35 Mary Augusta Clark

1935-37 George S. Stevenson, M.D.

* Deceased
1943-44 Milton E. Kirkpatrick, M.D.

Treasurer

1937-40 George S. Stevenson, M.D. 1940-43 Milton E. Kirkpatrick, M.D. 1944-48 James M. Cunningham, M.D.

Secretary

1937-43 Norvelle C. LaMar, M.D. 1944-48 Nina Ridenour, Ph.D.

\section{Councilors}

1924-26 V. V. Anderson, M.D.

1926-28 William Healy, M.D.

1928-30 Karl A. Menninger, M.D.

1930-31 Augusta F. Bronner, Ph.D.

1931-32 David M. Levy, M.D.

1932-33 Augusta F. Bronner, Ph.D.

1932-33 Ralph P. Truitt, M.D.

*1932-34 Herman M. Adler, M.D.

1933-35 George J. Mohr, M.D.

*1933-34 Ira S. Wile, M.D.

1934-35 Frederick H. Allen, M.D.

1934-36 Herbert E. Chamberlain, M.D.

1935-36 Mary Augusta Clark

1935-37 E. Van Norman Emery, M.D.

1936-38 Mildred C. Scoville

1936-37 Ralph P. Truitt, M.D.

1937-38 Edgar A. Doll, Ph.D.

1937-38 Harry M. Tiebout, M.D.

\section{Directors}

1938-39 Harry M. Tiebout, M.D.

1938-39 George J. Mohr, M.D.

1938-40 S. Spafford Ackerly, M.D.

1939-40 Frank J. O'Brien, M.D.

*1939-41 Jacob Kasanin, M.D.

1940-41 Samuel J. Hartwell, M.D.

1940-42 Simon H. Tulchin

1941-42 Paul L. Schroeder, M.D.

1941-42 Everett Kimball, Ph.D.

1942-43 Jacob Kasanin, M.D.

1942-43 Carmelite Janvier

1942-44 Katharine M. Wickman

1943-44 Henry C. Schumacher, M.D.

1943-44 Claudia Wannamaker

1943-46 H. S. Lippman, M.D.

1944-46 George H. Preston, M.D.

1944-47 Marian McBee

1946-47 Norvelle C. LaMar, M.D.

1946-48 S. Harcourt Peppard, M.D.

1947-48 Spafford Ackerly, M.D.

1947-49 George E. Gardner, M.D.

\section{Editor}

*1924-26 Herman M. Adler, M.D. 1926-27 Karl A. Menninger, M.D.

*1927-30 Herman M. Adler, M.D.

1930-51 Lawson G. Lowsey, M.D. 


\section{President-Elect}

1941-42 Henry C. Schumacher, M.D.

1942-43 George H. Preston, M.D.

1943-44 Norvelle C. LaMar, M.D.
1944-46 S. Spafford Ackerly, M.D.

1946-47 Milton E. Kirkpatrick, M.D.

1947-48 S. J. Beck, Ph.D.

MEETING PIACES OF THE A.O.A.

Organization Meeting, January 13, 1924

Annual Meetings

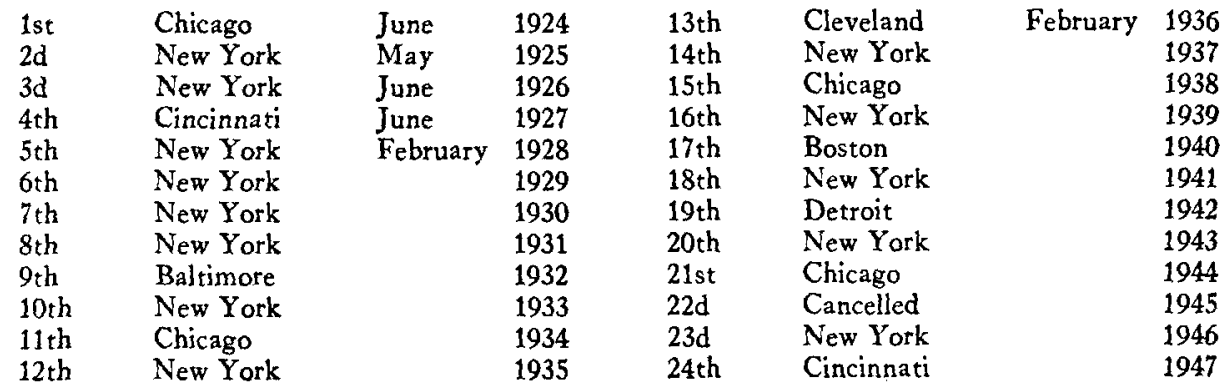

TWENTY-FIFTH ANNIVERSARY MEETING

New York, April 12, I3, I4, I948 